\title{
Theoretical Atomic Physics
}

Code Development II

\section{ACE: Another Collisional}

\section{Excitation Code}
R. E. H. Clark
J. Abdallah, Jr.
G. Csanak
J. B. Mann
R. D. Cowan

\section{DISCLAIMER}

\begin{abstract}
This report was prepared as an account of work sponsored by an agency of the United States Government. Neither the United States Government nor any agency thereof, nor any of their employees, makes any warranty, express or implied, or assumes any legal liability or responsjbility for the accuracy, completeness, or usefulness of any information, apparatus, product, or process disclosed, or represents that its use would not infringe privately owned rights. Reference herein to any specific commercial product, process, or service by trade name, trademark, manufacturer, or otherwise does not necessarily constitute or imply its endorsement, recommendation, or favoring by the United States Government or any agency thereof. The views and opinions of authors expressed herein do not necessarily state or reflect thoue of the United States Government or any agency thereof.
\end{abstract}

\section{MASTER}




\title{
THEORETICAL ATOMIC PHYSICS CODE DEVELOPMENT II ACE: ANOTHER COLLISIONAL EXCITATION CODE
}

\author{
by \\ R. E. H. Clark, J. Abdallah, Jr., G. Csanak, \\ J. B. Mann, and R. D. Cowan
}

\begin{abstract}
A new computer code for calculating collisional excitation data (collision strengths or cross sections) using a variety of models is described. The code uses data generated by the Cowan Atomic Structure Code or CATS for the atomic structure. Collisional data are placed on a random access file and can be displayed in a variety of formats using the Theoretical Atomic Physics Code or TAPS. All of these codes are part of the Theoretical Atomic Physics code development effort at Los Alamos.
\end{abstract}

\section{INTRODUCTION}

Collisional excitation cross sections or collision strengths for electron impact on atoms and ions are needed to model a non-Local Thermodynamic Equilibrium (nonLTE) plasma. As more computer codes become available to model such plasmas, the demand for the cross-section data increases. Plasma modelers are often faced with either no data or data from exceedingly simple models such as semi-empirical formulas or the plane-wave-Born approximation (PWBA). Frequently there is a need for a more sophisticated calculation of the cross-section data. Furthermore, it is desirable to have a fairly complete set of data and to have the cross-section data consistent with other atomic data such as energy levels and oscillator strengths. It is useful to have the computer code that is capable of generating the collisional data be easy to use and be accessible to a variety of users.

A new collisional excitation code called $A C E$, meeting all of these requirements, has been developed within the framework of the Theoretical Atomic Physics code development effort at Los Alamos. ${ }^{1-3}$ The main purpose of the code is to perform distorted wave approximation (DWA) calculations using J. B. Mann's method. ${ }^{4}$ 
For comparison purposes, Coulomb functions can also be used as in a code from G. Csanak, et al. ${ }^{5}$ First order many body theory ${ }^{6,7}$ calculations can also be performed in the code. PWBA calculations as described by Cowan ${ }^{8}$ for example. can also be done.

Atomic structure data needed for the collisional calculation are read from files produced by the CATS code. ${ }^{1}$ Values for all adjustable parameters are set to default values with commands available to change the values; thus, the code is easy to use. Data from the calculations are stored in the PARADISE ${ }^{9}$ format which is a random access binary file with a directory section that allows easy location of any data on the file. The directory is based on keywords that are descriptive of the stored data. The Appendix lists the data and associated keywords produced by ACE. Library routines exist for accessing the data easily. The TAPS code ${ }^{2}$ can be used to access the data for viewing at the user's terminal. Data are also written to a BCD file formatted for use with J. Abdallah's CURVES code, ${ }^{10}$ a general-purpose graphics code.

In the following sections, we describe the procedure for running $A C E$, give a brief description of the physics in the code, and give some examples of calculations performed with ACE.

\section{RUNNING ACE}

\section{A. Setting Up the Atomic Structure Files}

The ACE code reads-in all atomic structure information from files generated by the CATS code. ${ }^{1}$ Both executable codes, ACE and CATS, are available from the Common File System (CFS) at Los Alamos under the root node /TAPS. The codes are unclassified and do not require a password for access.

Frequently, collisional calculations involve a set of transitions from one group of levels to another. Often, transitions within each group are not needed. Thus, the default in $\mathrm{ACE}$ is to attempt to read atomic structure data for an initial set of levels from a file named groupl and for a final set of levels from a file named group2. These files can be generated by CATS using two separate runs, one with the OPEN GROUP1 command, the second using OPEN GROUP2. If desired, both sets of levels can be included in one structure file, and the ACE command FILES (described more fully below) can be used to override the default names groupl and group2. In any case, it will be assumed that either one or two atomic structure files are available for $A C E$.

\section{B. ACE Commands}

$\mathrm{ACE}$ is an interactive code. After typing $\mathrm{ACE}$ followed by a carriage return, the user can type commands. Table I gives a list of commands available in ACE. The following section describes each command in detail. The commands are sorted into categories based on their function. The categories are $\mathrm{I} / \mathrm{O}$, display, selection. grids. physics options, and code action. 
TABLE I. ACE COMMANDS

\begin{tabular}{|c|c|c|c|}
\hline FILES & fname 1 & \multicolumn{2}{|l|}{ (fname?) } \\
\hline OLTFILE & fname & & \\
\hline INFILE & fname & & \\
\hline ION & 2 & ion & seq \\
\hline SEQ & $i$ & & \\
\hline GOS & $\mathrm{T} / \mathrm{F}$ & & \\
\hline LEVELS & & & \\
\hline TERMS & & & \\
\hline CFG & & & \\
\hline SETL & $i_{1}$ & $i_{2}$ & \\
\hline SETT & $i_{1}$ & $i_{2}$ & \\
\hline SETC & $i_{1}$ & $i_{2}$ & \\
\hline SETLE & $i_{1}$ & $i_{2}$ & $i_{3}$ \\
\hline $\mathrm{X}$ & $x_{1}$ & $x_{2}$ & $x_{3}$ \\
\hline XLOG & $\mathrm{nx}$ & $x_{L}$ & $x_{u}$ \\
\hline XLIN & $\mathrm{nx}$ & $x_{L}$ & $\mathrm{x}_{\mathrm{u}}$ \\
\hline ELONITS & option & & \\
\hline$E$ & $e_{1}$ & $e_{2}$ & $e_{3}$ \\
\hline ELOG & $\mathrm{n}_{e}$ & $\mathrm{e}_{L}$ & $\mathrm{e}_{\mathbf{u}}$ \\
\hline ELIN & $\mathrm{n}_{e}$ & $e_{L}$ & $e_{\mathbf{u}}$ \\
\hline THETA & $a_{1}$ & $a_{2}$ & $\ldots$ \\
\hline THETALIN & $\mathrm{n}$ & $\mathrm{a}_{L}$ & $a_{u}$ \\
\hline THETALOG & $\mathrm{n}$ & $a_{L}$ & $a_{u}$ \\
\hline LMAXX & $\ell_{\max }$ & & \\
\hline BOEND & option & & \\
\hline FREE & option & & \\
\hline POT & option & & \\
\hline LNI & option & & \\
\hline PWB & $\mathrm{T} / \mathrm{F}$ & & \\
\hline DCS & $T / F$ & & \\
\hline AVPHASE & $T / F$ & & \\
\hline HELP & & & \\
\hline GO & & & \\
\hline END & & & \\
\hline
\end{tabular}

Any command line requiring a list of options can be continued by placing an ampersand $(\&)$ as the last character on a line. Simply continue the input (without retyping the command itself) on the next line. This action may be repeated as often as necessary.

For commands with options, the current option setting can be displayed by typing the command name alone. For example, typing FILES will cause ACE to display the currently defined files for atomic structure data. Exceptions to this are the commands INFILE, XLOG, and XLIN. 
1. I/O Commands. To specify the file names containing the atomic structure information. use the command

FILES fname1 (fname2)

where fname 2 is omitted if all structure data are contained on file fnamel. The default file names are groupl and group2. When ACE begins a run, it automatically checks the user's local file space for files groupl and group2. If they are present. the directories are read and all ion stages are identified (more than one ion stage can exist on a file).

All ion stages common to groupl and group2 are identified, and the first common stage found is selected for use in the calculation (any other stage can be selected with the ION command). If no common stages are iound. a message is displayed. This procedure is repeated whenever the FILES command is used with two file names. If only one file name is given, the first ion stage present on the file is selected.

Output from $A C E$ is normally written to a PARADISE ${ }^{9}$ formatted file. The default file rame is adam. The name can be changed with the command

\section{OUTFILE fname}

where fname is the desired file name. If the letter $f$ is used, this letter is interpreted as a command to turn of the PARADISE file writing completely.

$A C E$ normally runs interactively; however, commands can be read from a file generated with a text editor. The command

\section{INFILE fname}

will switch control to commands in the file given by fname. This command may be given on the execute line:

\section{ACE INFILE fname}

causing the code to read all commands from the file fname instead of from the terminal.

If more than one set of structure data appears on a file from CATS (or if there is more than one common ion stage on two files), an ion stage can be selected using

$$
\text { ION } z \text { ion seq }
$$

where $z$ is the atomic number, ion is the ionicity (in spectroscopic notation: 1 for neutral, 2 for once ionized. otc.), and seq is a sequence number. It is possible to have two different versions of .le same $z$ and ion values on a file, each with a different sequence number.

When results of a calculation are put on the output file. the sequence number is normally set to 1 . The output sequence number can be set to any value using the command 


\section{SEQ i}

where $i$ is the desired sequence number.

For every transition. ACE calculates generalized oscillator strengths for use in a PWBA calculation. The generalized oscillator strengths can be written as a function of momentum. $K$, to a file named plotgos formatted for the CLRVES code $^{10}$ using the command

$$
\text { GOS option }
$$

where option may be $t$ to generate the output file or $f$. which is the default. to turn off the file generation.

2. Display Commands. After an ion stage has been selected. some information from the structure data can be displayed. The command

\section{LEVELS}

will display a list of levels in the problem. Similarly, the commands

TERMS

\section{CFG}

list the LS terms and configurations, respectively.

3. Selection Commands. It is possible to select a subset of all possible . transitions. The command

$$
\text { SETL } i_{1} i_{2}
$$

will cause calculations of collision strengths between levels $i_{1}$ and $i_{2}$ to be performed when the GO command is executed. All other transicions will be skipped. The values of $i_{1}$ and $i_{2}$ correspond to the indexing of levels displayed with the LEVELS command. Either $i_{1}$ or $i_{2}$ can be an asterisk $\left({ }^{*}\right)$, which means all levels. Thus. the command

\section{SETL $1 *$}

selects transitions from level 1 to all other levels. The command

$$
\text { SETL * * }
$$

selects all transitions.

The commands

$$
\begin{array}{ll}
\text { SETT } i_{1} & i_{2} \\
\text { SETC } i_{1} & i_{2}
\end{array}
$$


are identical to SETL $i_{1} i_{2}$ except that they operate on terms and configurations. respectively. We note that $\mathrm{ACE}$ will calculate level-to-level transitions within a term or configuration. Thus, in the SETT and SETC commands, $i_{2}$ may equal $i_{1}$. Also, in those commands, using the asterisk for $i_{1}$ or $i_{2}$ means ACE will go over all terms or configurations. Thus, the command

\section{SETC $1^{*}$}

will cause $A C E$ to calculate level-to-level transitions within configuration 1 , as well as those from configuration 1 to other configurations. Setting $i_{1}$ or $i_{2}$ to \# in the SETT or SETC command will skip level-to-level transitions within a term or configuration, respectively. Thus, the command

\section{SETC $1 \#$}

would calculate transitions from configuration 1 to other configurations and would skip to level-to-level transitions within configuration 1.

The default is for $A C E$ to calculate all level-to-level transitions from configuration 1 to all other levels, i.e., $i_{1}=1$ and $i_{2}=*$.

Occasionally, the energy levels calculated from CATS may not be as close as desired to observed values. The desired values can be read in after the command

\section{SETLE $i_{1} i_{2} \quad \ldots$}

is given. ACE will go through the list $i_{1}, i_{2}, \cdots$ of levels one at a time, displaying the value from the file and asking for a new value. The user may type in a new value or give a carriage return to leave the value unchanged. The asterisk $\left(^{*}\right)$ in the SETLE command will cause ACE to go through every level in the problem. Note that changing the energy values will result in an inconsistency between the energies and the wave functions.

4. Grid Commands. A default grid for the incident electron energy of five points logarithmically spaced from 1.01 to 20 in threshold $(x)$ units is used in ACE. The grid can be changed with any of three commands.

The command

$$
\begin{array}{llll}
\mathrm{X} & \mathrm{x}_{1} & \mathrm{x}_{2} & \cdots
\end{array}
$$

allows the user to type in $x$ values individually. Up to $100 x$ values may be used. An input line can be continued by making the last character on the line an ampersand (\&).

The commands

$$
\begin{array}{llll}
\text { XLOG } & n x & x_{L} & x_{u} \\
\text { XLIN } & n x & x_{L} & x_{u}
\end{array}
$$


set up grids of $n \times x$ values from $x_{L}$ to $x_{u}$ with logarithmic and linear spacing. respectively. To display the current grid. type $x$ with no values after it.

The user can select units for the energy grid using the command

\section{ELNITS option}

where option may be $\mathrm{x}$, ev, ryd, or au for threshold units, electron volts. rydbergs or atomic units (hartrees), respectively. Using any of the $x$-grid commands ( $X$. XLOG, or XLIN) automatically resets EUNITS to $\mathrm{x}$.

Energy grids in units other than threshold units can be entered one at a time using the

$$
\begin{array}{llll}
E & e_{1} & e_{2} & \cdots
\end{array}
$$

command. Logarithmic and linear grids can be generated using the

$$
\begin{aligned}
& \text { ELOG } n_{e} \quad e_{L} e_{v} \\
& \text { ELIN } n_{e} \quad e_{L} e_{u}
\end{aligned}
$$

commands, respectively.

The user has control of the angular grid used for calculation of differential cross sections and coherence parameters. The commands

$$
\begin{array}{lllll}
\text { THETA } & a_{1} & a_{2} & a_{3} & \cdots \\
\text { THETALIN } & n & a_{L} & a_{v} \\
\text { THETALOG } & n & a_{L} & a_{v}
\end{array}
$$

are analogous to the energy grid commands except that they work on angles. A default grid using every $10^{\circ}$ from $0^{\circ}$ to $180^{\circ}$ is used if no angles are specified by the user.

5. Physics Options. The maximum $\ell$ value in the summation appearing in the Kummer transformation ${ }^{11}$ (see Section III, Eq. 1) has the default value of 20 . This value can be changed with the command

$$
\text { LMAX } \ell_{\max }
$$

where $\ell_{\max }$ is the desired maximum $\ell$ value.

The default $A C E$ calculation is a distorted wave approximation (DWA) ${ }^{4}$ calculation using bound state wave functions from CATS. Several changes to these defaults can be made.

The bound state wave function can be changed using the command

$$
\text { BOLND option }
$$


where option can be hyd for a set of hydrogenic orbitals or hf for the (normally Hartree-Fock-relativistic) wave functions from CATS. When hyd is selected. the energy levels and mixing coefficients are still read from the CATS file: only the radial wave functions are changed.

The command

\section{FREE option}

is used to switch from a free electron wave function calculated in the DWA (option is $\mathrm{dw}$; this is the default) to aroulomb free-electron wave function (option is coul).

The potential used in the DWA calculation can be changed using the command

$$
\text { POT option }
$$

The option mann sets the potential to the DWA form of Mann, ${ }^{4}$ while the option fomb uses the first-order many-body theory form. ${ }^{6,7}$

In normal operation, ACE unitarizes the rho matrix. ${ }^{4}$ This option can be controlled through the command

\section{UNI option}

where the option $t$ enforces unitarization, and $f$ turns it off.

The command

\section{PWB option}

allows the user to have a PWBA calculation if option is $t$. The default for this option is $f$, which means no PWBA calculation is performed.

The command

\section{DCS T/F}

is used to generate the complex scattering matrix from which differential cross sections (DCS) and coherence parameters (CP) can be calculated. The scattering amplitude is stored on the PARADISE file and the DCS and CP values are calculated in the TAPS code. ${ }^{2}$ The default is $F$, so that the scattering amplitude is not calculated.

The command

\section{AVPHASE T/F}

controls the calculation of the free electron phase for use in the calculation of the scattering amplitude. $T\}$ default value of $T$ causes an average over mixed states to be used (see Sec. If details) while the value of $F$ causes the phase to be calculated using ony the dominant configuration.

6. Code Action Commands. Information about a specific command can be obtained by typing 


\section{HELP command}

where command is the name of the command for which information is sought. If no command name is given, i.e., HELP is typed alone, a list of available commands will be displayed.

To start a calculation, use the command

GO.

The command

END

concludes the ACE program.

\section{Output}

The primary output from ACE is a PARADISE file. This file contains the atomic structure information used by $A C E$, the incident electron energy grid, and the fine structure level-to-level collision strengths. Subroutines ${ }^{9}$ exist to extract these data for use in other codes directly. The TAPS code ${ }^{2}$ can be used to view the results at the terminal, make plots, or send results to a print file. TAPS can also perform sums to obtain term-to-term collision strengths as well as configuration-to-configuration collision strengths. The results can also be displayed in the form of cross sections. See the Appendix for details of the information stored by ACE on the PARADISE file.

$A C E$ also generate: a text file with the name tape8. Information from the Kummer transformation (see Section III) is contained on this file. Specifically, at each energy, the total collision strength, sum of partial PWBA collision strengths, and the total PWBA collision strength is printed. Thus, the user car readily see how closely the collision strength is converged for each energy. If th': two PWBA collision strengths (sum of partial waves and the total) do not agree to within $1 \%$ at the lowest energy, information is written to a file called acelog, and a message is displayed at the terminal if the user is in interactive mode or to the file outfile if the user employs the INFILE option.

\section{PHYSICS ISSUES}

$A C E$ uses the DWA method of Mann. ${ }^{4}$ We will not repeat the DWA formalism here, but will comment on features in ACE that differ from Mann's codes.

ACE has been written to calculate collision strengths between fine structure levels using the level-to-level transition energy rather than the term-to-term transition energy. This means that the free-electron wave functions are calculated with a different $\mathrm{K}^{2}$ for each level-to-level transition. In practice, to save time, calculations are performed at three different transition energies for each configuration-changing transition, the three energies representing the largest, smallest, and an intermediate 
level-to-level transition energy. The configuration-to-configuration matrix elements are then calculated and fitted to the transition energy. The fit is $u$ s. to reconstruct the matrix for a particular level-to-level transition. This procedure works quite well. A check on this procedure is the comparison of the sum of partial PWBA collision strengths calculated using this procedure to the total PWBA collision strength calculated from the generalized oscillator strength. Near threshold, where the partial wave calculation is well converged, the two usually agree to $\sim 0.1 \%$ if the spread in transition energies is not large.

$A C E$ includes configuration interaction and intermediate coupling mixing in the target state in much the same way as Mann does. ${ }^{4}$ However, in the recoupling to fine structure levels, ACE uses the pair-coupling scheme of Saraph, ${ }^{12}$ which is implemented exactly as by Clark. ${ }^{13}$ This effectively replaces each $9-\mathrm{j}$ symbol with two 6 -j symbols.

In order to calculate a well-converged collision strength at any energy with a relatively small number of partial waves, the Kummer transformation ${ }^{10}$ is used:

$$
\Omega=\Omega^{P W B}+\sum_{\ell=0}^{\ell_{\max }}\left(\Omega_{\ell}^{D W}-\Omega_{\ell}^{P W B}\right),
$$

where $\Omega^{P W B}$ is the total PWBA collision strength calculated from the generalized oscillator strength, $\Omega_{\ell}^{D W}$ is the partial wave DWA collision strength, and $\Omega_{\ell}^{P W B}$ is the calculated with the same subroutines as $\Omega_{\ell}^{D W}$ using Bessel functions in place of the free-electron wave function calculated from the distorted wave equations. Agreement of $\sum_{\ell} \Omega_{\ell}^{P W B}$ with $\Omega^{P W B}$ at low energy is a valuable check on those subroutines. Equation 1 is obviously valid when $\ell_{\max } \rightarrow \infty$ because then $\sum_{\ell} \Omega_{\ell}^{P W B} \rightarrow \Omega^{P W B}$. At low energies, both $\Omega^{P W B}$ and $\Omega_{\ell}^{P W B} \rightarrow 0$. Furthermore, the DW collision strength converges at quite small values of $\ell$ at low energy; i.e., $\Omega_{\ell}^{D W} \rightarrow 0$ for $\ell \sim 10-20$. As the energy increases, the number of $\ell$ values needed for convergence of $\Omega^{D W}$ increases but, simultaneously, the PWBA calculation approaches the DWA calculation. Thus, at moderate values of $\ell, \Omega_{\ell}^{P W B} \simeq \Omega_{\ell}^{D W}$ and, for large $\ell,\left(\Omega_{\ell}^{D W}-\Omega_{\ell}^{P W B}\right) \rightarrow 0$. At very high energy, $\Omega^{P W B} \simeq \Omega^{D W}$. In practice, it appears that, with $\ell_{\max }=20$, the result given by Eq. (1) is accurate to better that $5 \%$ at all energies and usually is considerably better than that.

Several choices are available in ACE for radial wave functions. Normally, the bound state wave functions are read from a file produced by the CATS code. These wave functions are normally the Hartree-Fock-Relativistic (HFR) functions of Cowan. ${ }^{8} \mathrm{ACE}$ has the option of replacing these wave functions with hydrogenic radial functions calculated with an effective nuclear charge of

$$
Z_{\text {eff }}=Z-N+1,
$$

where $\mathrm{Z}$ is the atomic number and $\mathrm{N}$ is the number of bound electrons. The freeelectron wave function is normally calculated using the distorted wave equations. ACE has an option to change to a first-order many-body theory (FOMBT) ${ }^{6,7}$ calculation. In practice, this simply changes the potential used in the calculation of the outgoing electron from the excited state potential to the initial target state 
potential. It is also possible to use Coulomb functions for the free-electron wave functions. Also, a PWBA calculation can be requested. Thus, one potential usefulness of the $A C E$ code is an easy way to compare the effects of different radial wave functions on the collision strength calculation.

In obtaining the complex scattering amplitude for use in calculating differential cross sections (DCS) and coherence parameters (CP), the recoupling scheme of Inal and Dubau ${ }^{14}$ is used. The phase of the free electron is normally calculated using the mixing coefficients and the phases of the free electron wave functions calculated from the individual single configuration target states:

$$
\delta_{j}=\sum_{i=1}^{N_{c o n f i g}} a_{i j}^{2} \delta_{i},
$$

where the $a_{i j}$ is the mixing coefficient, the $\delta_{i} \mathrm{~s}$ are the phases calculated from single configurations and $\delta_{j}$ is the phase for the mixed state. The user can cause ACE to use only the phase from the single configuration making the dominant contribution by using the AVPHASE command. The two methods normally make insignificant differences in the DCS and CP calculations.

Finally, we mention that most of the arrays in ACE use S. W. White's memory manager. ${ }^{15}$ Thus, the main limitation on complexity of a problem is the size of the computer, not dimension statements in the code. As larger computers become available, bigger problems can be run with no change in ACE.

\section{EXAMPLES}

To illustrate the use of ACE, we consider the case of NIII. We include six configurations: $2 s^{2} 2 p, 2 s 2 p^{2}, 2 p^{3}, 2 s^{2} 3 s, 2 s^{2} 3 p$, and $2 s^{2} 3 d$. The input file for CATS is shown in Fig. 1. We have named the file for the structure data n3. We have also requested PWBA collision strengths. Figure 2 shows a printout from the terminal during the CATS run.

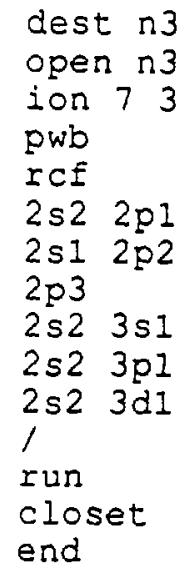

Fig. 1. Listing of file $\mathrm{i}-$-input to CATS. 


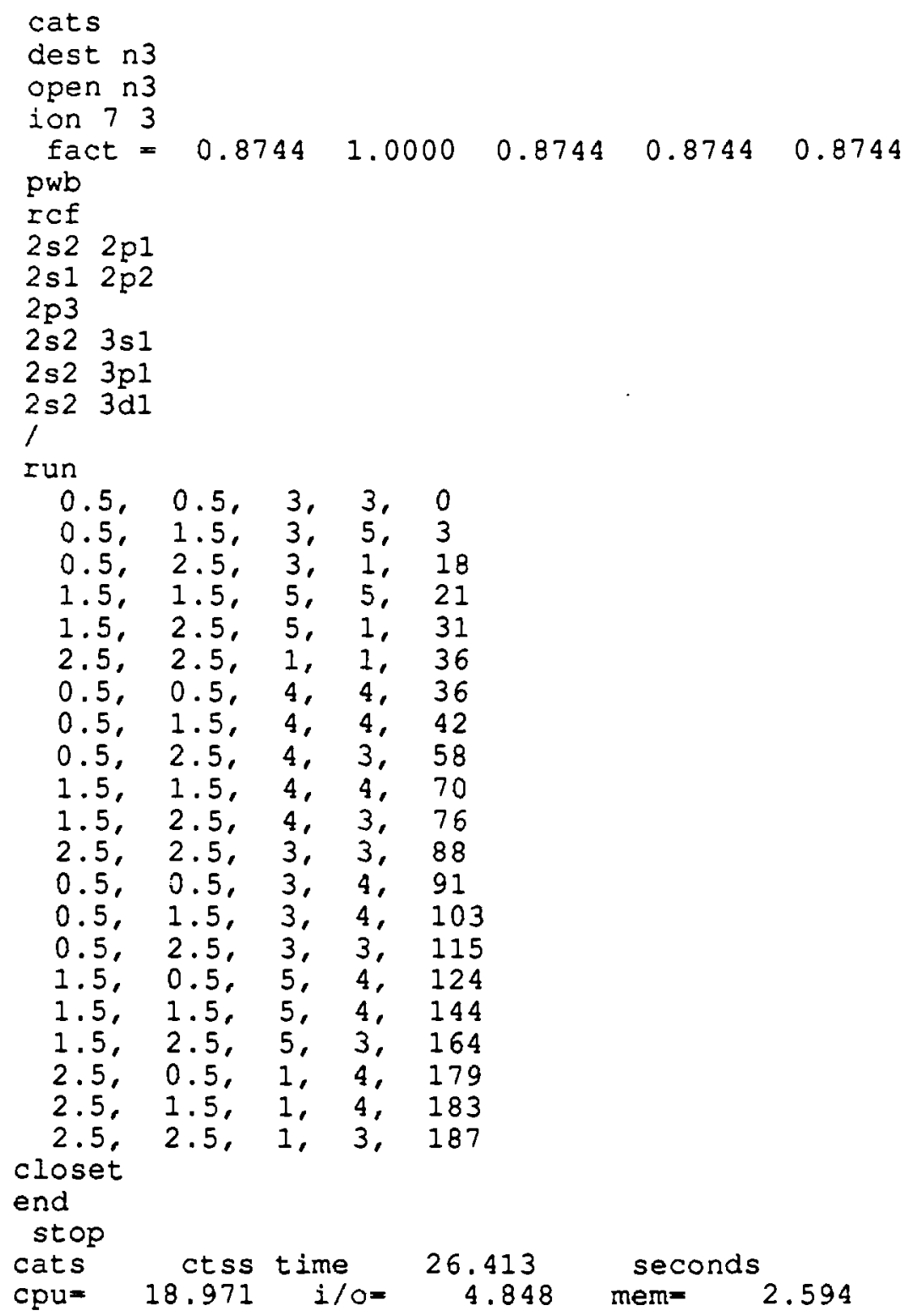

Fig. 2. Printout from terminal during CATS run. 
We next run $A C E$ on this case. Refer to Fig. 3 for a copy made from the terminal during the interactive run. Note the FILES command with the one file name. $\mathrm{n} 3$, which causes ACE to search that file for structure data. Since there is only one entry on the file, the ION command is not needed. The CFG command lists the configurations. We use the SETC command to request transitions from all levels of configuration 1 (the $2 s^{2} 2 p$ configuration) to all levels of all configurations (including configuration 1). We use the XLOG command to select 21 energy points logarithmically spaced between 1.01 and 1000 . The $X$ command with no options displays the energy grid. The GO command is then issued.

After completing the calculations, $\mathrm{ACE}$ prints a message that there are problems in the PWBA calculation for 20 transitions. The entire calculation took $950.6 \mathrm{CPC}$ seconds. There are 37 level-to-level transitiors at each of 21 energies. Thus, it took $A C E$ an average of 1.2 seconds per transition at each energy. This is typical of DWA calculations with this version of ACE. Potential speedup of the code is being investigated.

Figure 4 shows a listing of the file acelog. The transitions listed are all the ones with a disagreement of $1 \%$ or more between the sum of partial PWBA collision strength compared to the total PWBA collision strength at the lowest energy. We point out that most of the transitions have a discreparicy of less that $5 \%$, with the largest being less than $10 \%$. The cause of the discrepancy is the fitting of the configuration-to-configuration matrix elements as a function of transition energy as discussed in Section III. In the present case, the level-to-level transition energies range from $0.02 \mathrm{eV}$ for the $2 \mathrm{~s}^{2} 2 \mathrm{p}^{2} \mathrm{P}_{\frac{1}{2}}-2 \mathrm{~s}^{2} 2 \mathrm{p}^{2} \mathrm{P}_{\frac{3}{2}}$ transition (which $\mathrm{ACE}$ does calculate) to over $30 \mathrm{eV}$ for transitions to the levels of the $2 s^{2} 3 p$ configuration. This extremely wide range of transition energies caused the $\sim 5 \%$ errors in the PWBA collision strengths when the matrix was reconstructed from the fit. To check the effect on the total collision strength, ACE was rerun using the command SETL 2 * so that the level 1-2 transition was excluded. Comparison of these results to the original results is shown in Fig. 5. We note that there is little effect on any of the transitions. 


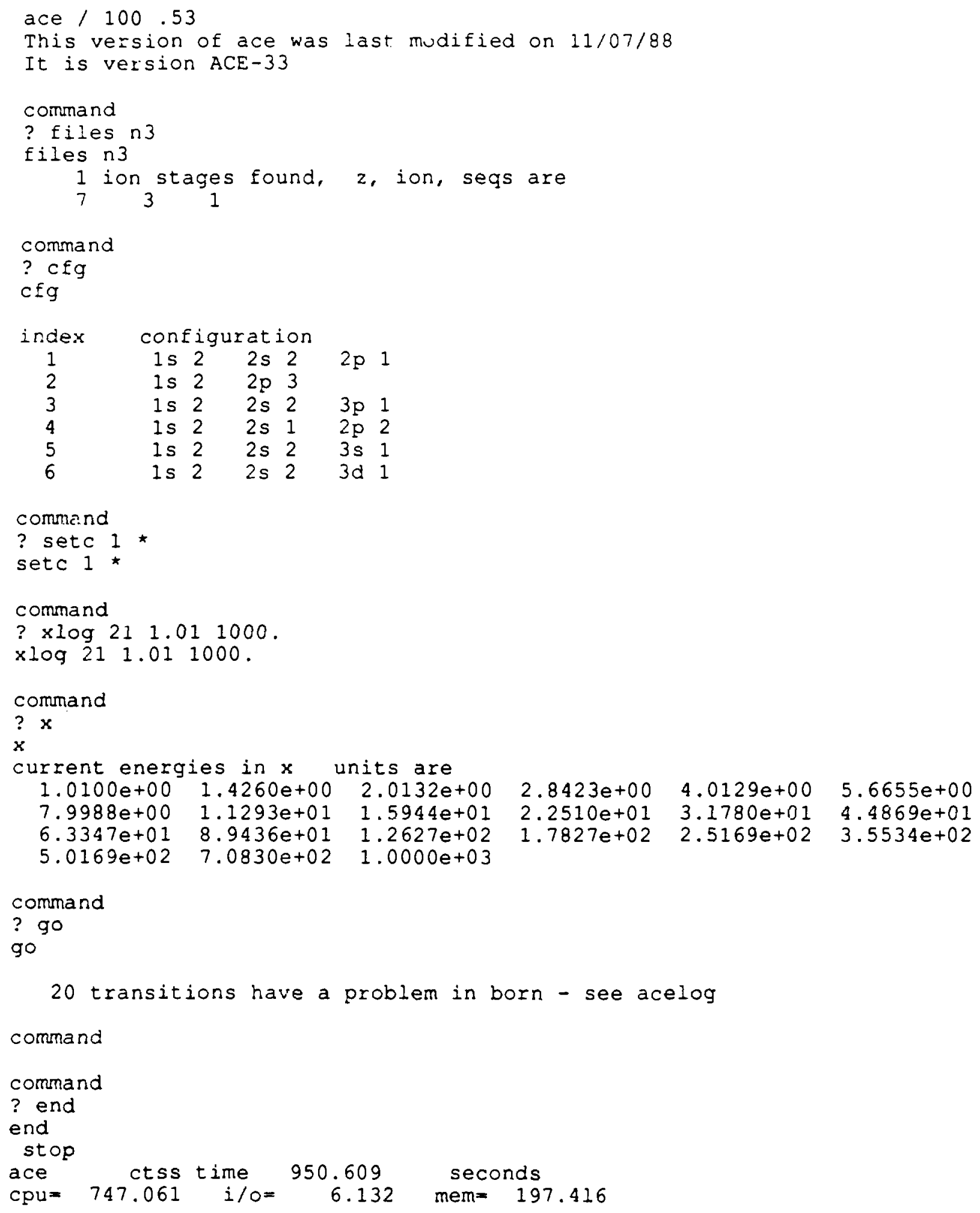

Fig. 3. Printout from terminal during $\mathrm{ACE}$ run. 


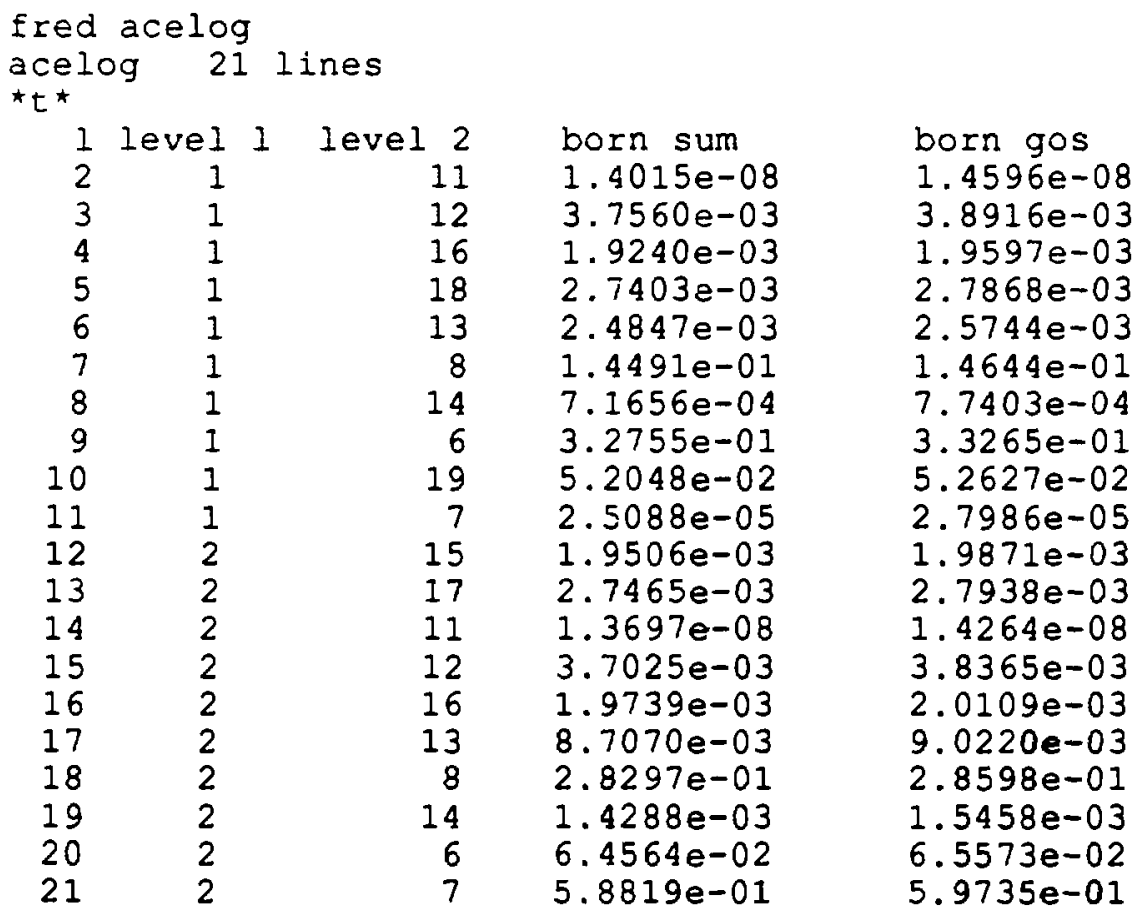

Fig. 4. Listing of acelog. 


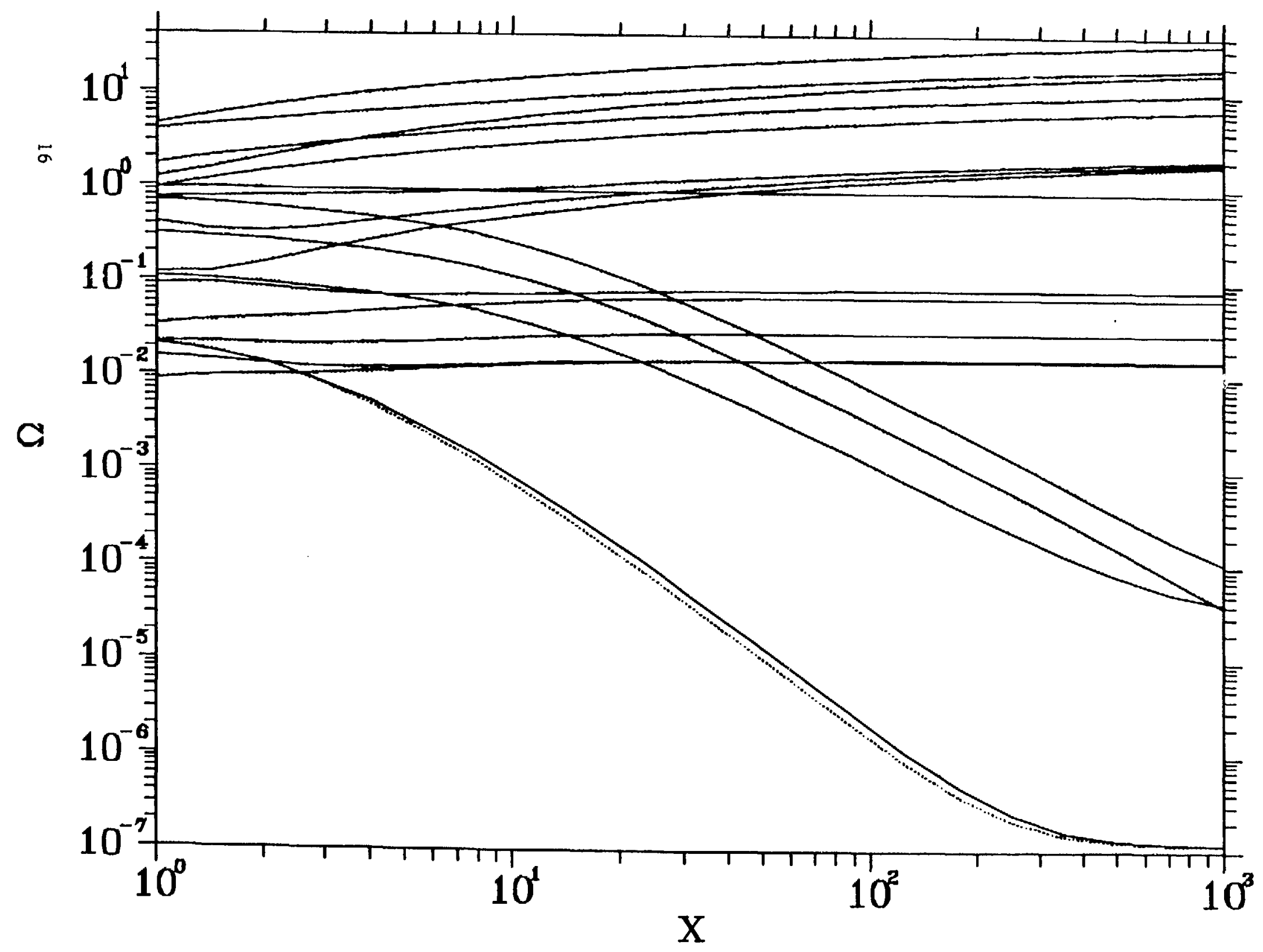

Fig. 5. Graphs of collision strengths $(\Omega)$ for transitions from level 2 to all other levels. Solid line from $A C E$ run, including all transitions; dotted line from $A C E$ run, excluding 
Since there are significant differences between the coding in $A C E$ and Mann's DWA codes, it is useful to compare ACE results to those published by Mann. ${ }^{4}$ Any significant differences could indicate problems with $A C E$. It is important to note that there can be some differences in the structure calculations. Cowan's codes have changed over the past 10 years. resulting in some differences in the calculated radial wave functions. Also, if the scale factors used are not identical, different mixing coefficients will result. CATS includes an algorithm to set scale factors based on atomic number and ion stage. These default scale factors sometimes give somewhat different results from Mann's choices of scale factors.

As an example, Fig. 6 shows collision strengths for the $2 s^{2}-2 s 2 p\left({ }^{3} P\right)$ transition in Fe XXIII. The circles are Mann's values, the crosses are from ACE using the default CATS run, and the triangles are from ACE after the srale factors in CATS had been adjusted to give close agreement with Mann's mixing coefficients. The default scale factors are 1.0 for the spin-orbit term and 0.9325 for all others. These were modified to 0.92 for the spin-orbit term and 0.82 for all others. Figure $T$ shows similar comparisons for the $2 \mathrm{~s}^{2}-2 \mathrm{~s} 2 \mathrm{p}\left({ }^{1} \mathrm{P}\right)$ transition. The excitation to the ${ }^{3} \mathrm{P}$ shows clearly the effect of the scale factors. In Fig. 7 , note that the maximum discrepancy between Mann's results and the ACE results occurs around $x=10$. This discrepancy is caused by the Kummer transformation, which gives best results at low energies and high energies. The worst results occur at intermediate energies where the DWA result is not yet very close to the PWBA result, but many partial waves are need d for convergence. In our example, there is a maximum error of $\sim 5 \%$ in this energy region. To show clearly that this is the explanation, we reran the $2 s^{2}-2 s 2 p^{1} P$ transition with $\ell_{\max }=30$ (instead of the default $\ell_{\max }=20$ ). The result is shown in Fig. 8, which shows the discrepancy at intermediate energies nearly gone.

We next show the effects of different wave functions on the collision strengths. Figure 9 compares the DW-HF (triangles) with the Coulomb hydrogenic (X's) and the PWB (boxes with X's) for the $2 s^{2}-2 s 2 \mathrm{p}^{1} \mathrm{P}$ transition in Fe XXIII. The Coulombhydrogenic calculation is in good agreement, as might be expected for this high stage of ionization. The PWB calculation is within $\sim 20 \%$ of the DW calculation for $x>2$.

In Fig. 10, a similar comparison is made for the $3^{2} \mathrm{~S}-3^{2} \mathrm{P}$ transition in Fe XVI. The plot symbols are as before, with Mann's results (circles) included for comparison. In this case, the Coulomb-hydrogenic calculation is considerably off. The results from $A C E$ are in excellent agreement with Mann's results. 


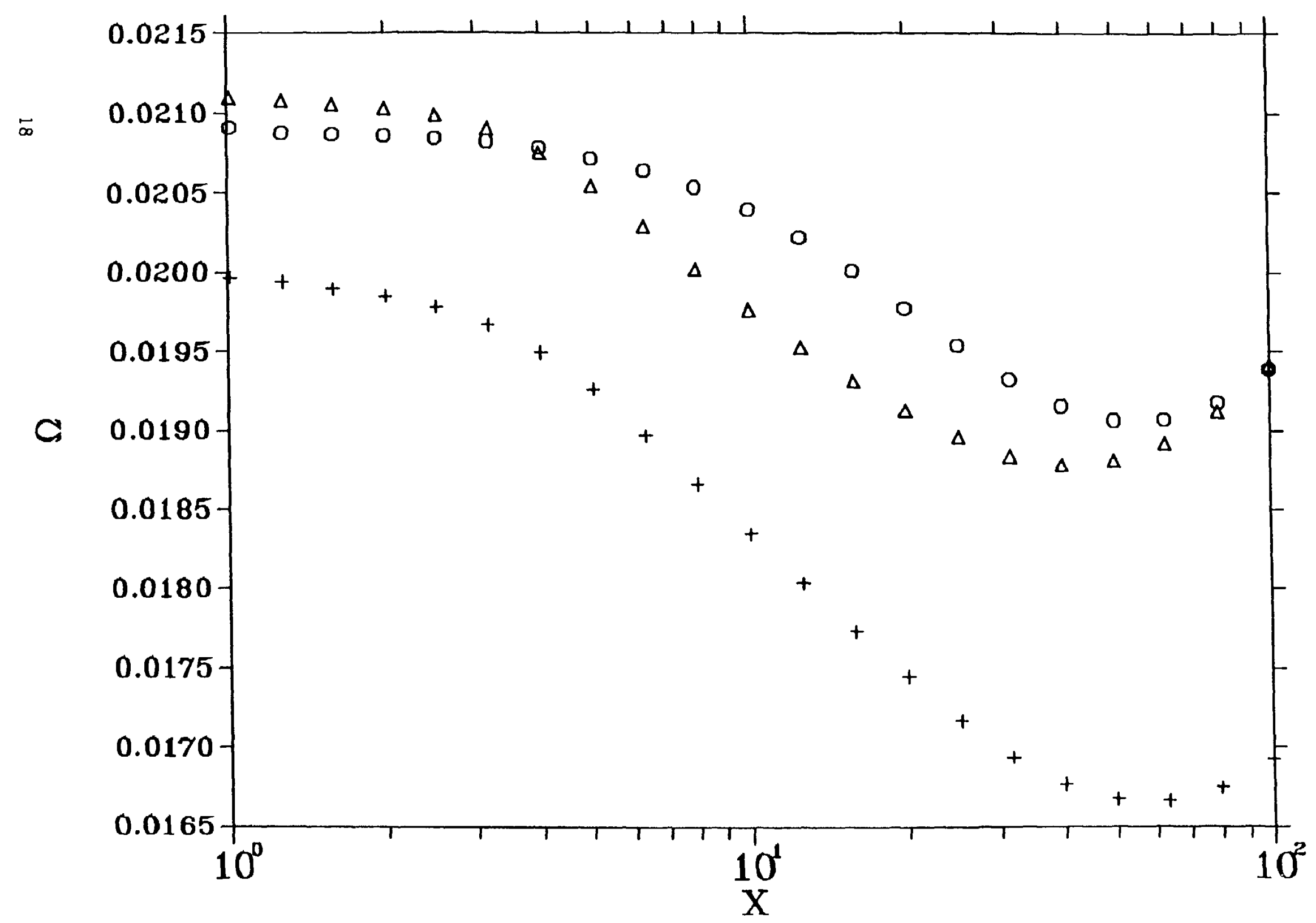

Fig. 6. Collision strengths $(\Omega)$ for the $2 \mathrm{~s}^{2}-2 \mathrm{~s} 2 \mathrm{p}^{3} \mathrm{P}$ transition in Fe XXIII. Circles represent results of Mann; triangles are results from ACE witl modified scale factors; crosse's inre from ACE with defiult scale fartors from CATS. 


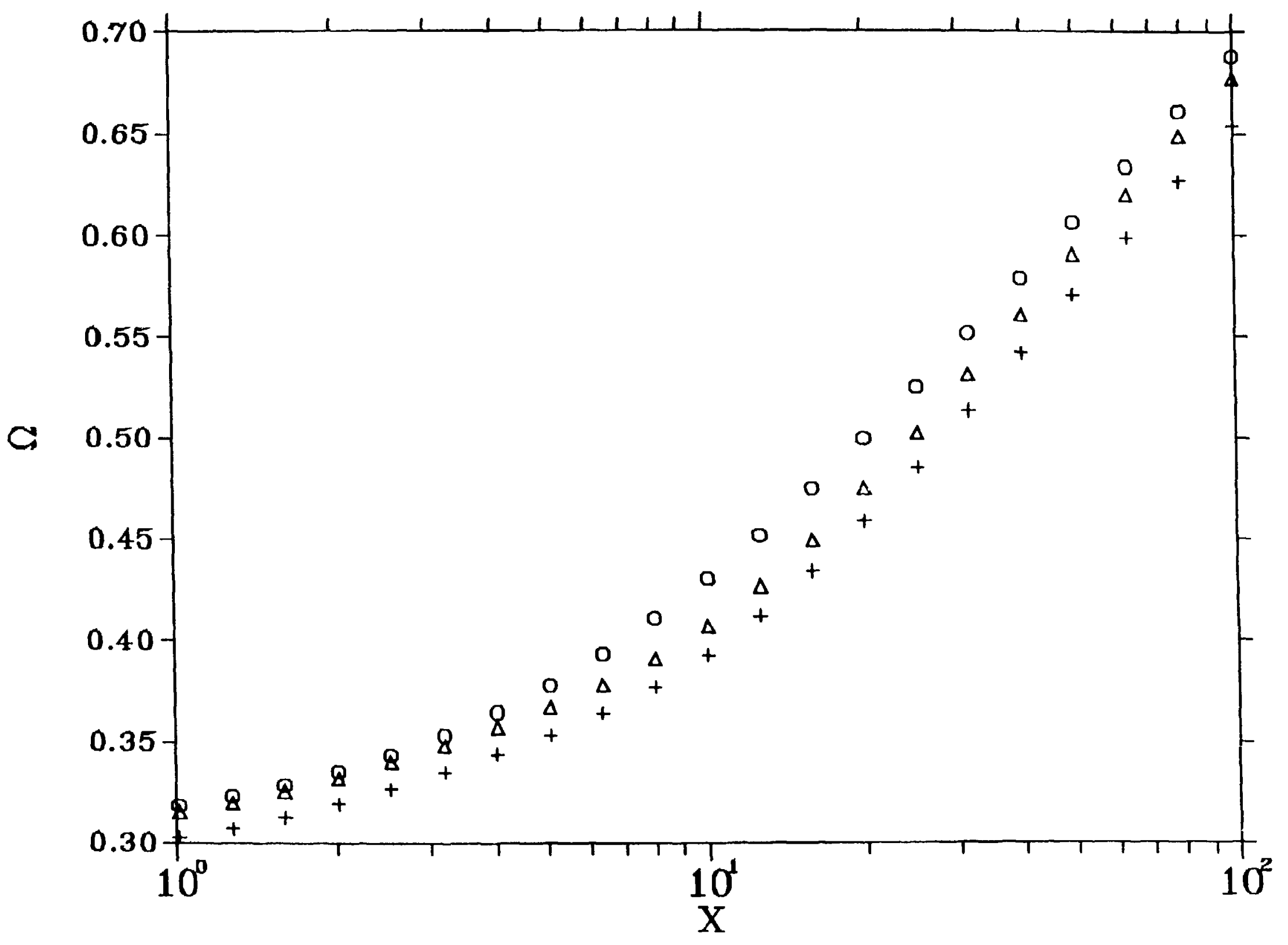

Fig. 7. Collision strengths $(\Omega)$ for the $\left.2 s^{2}-2 s 2_{p}\right)^{1} P$ transition in Fe XXIII. Circles represent results of Mann; triangles are results from ACE with modified seale fartors; crosses are fron ACE with refault scale factors from C'A'TS. 


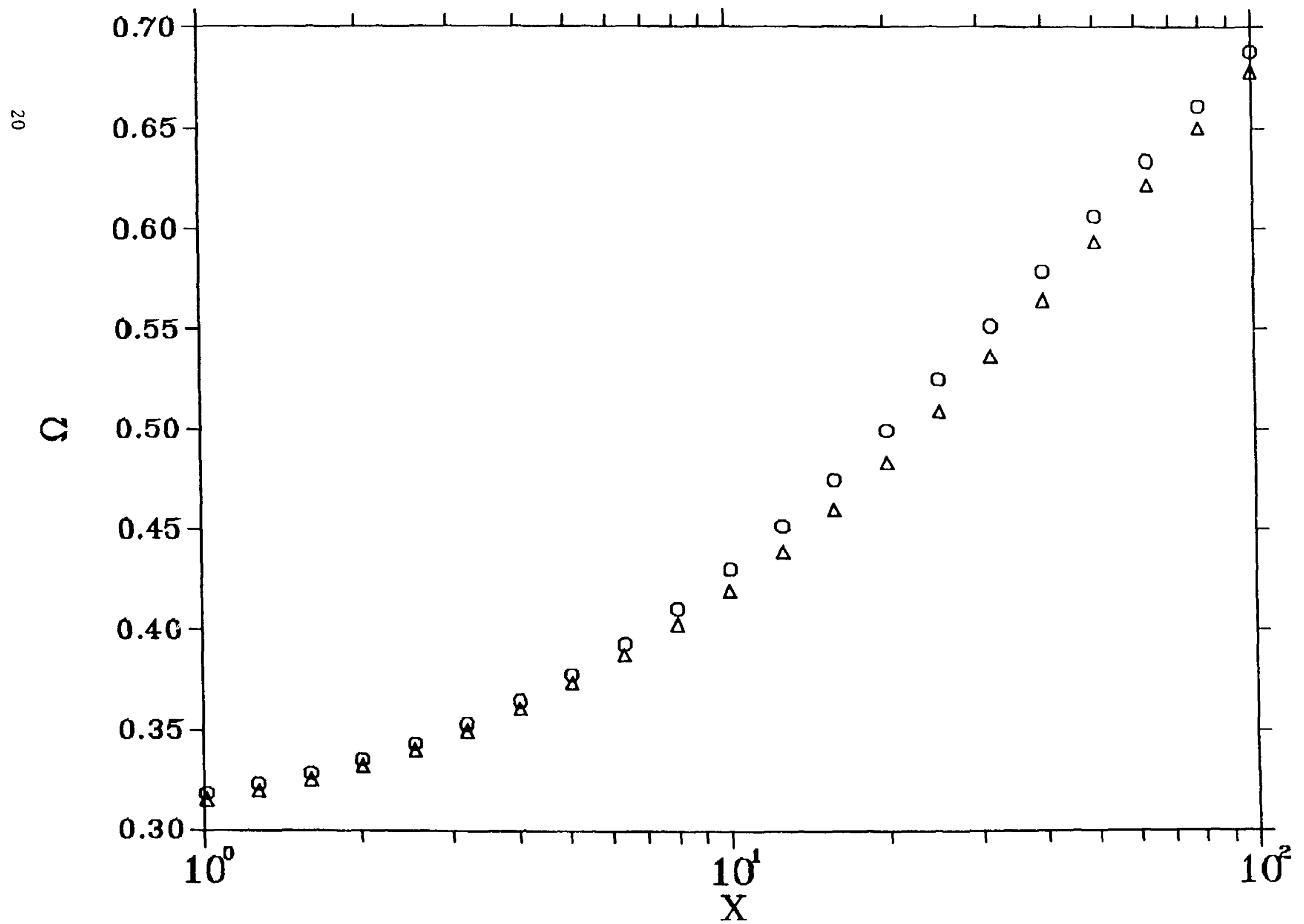

Fig. 8. Collision strengths $(\Omega)$ for the $2 s^{2}-2 s^{2} p^{1} P$ transition in Fe XXIII. Circles represent results of Mann; triangles represent ACE results with moditied scale factors and

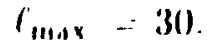




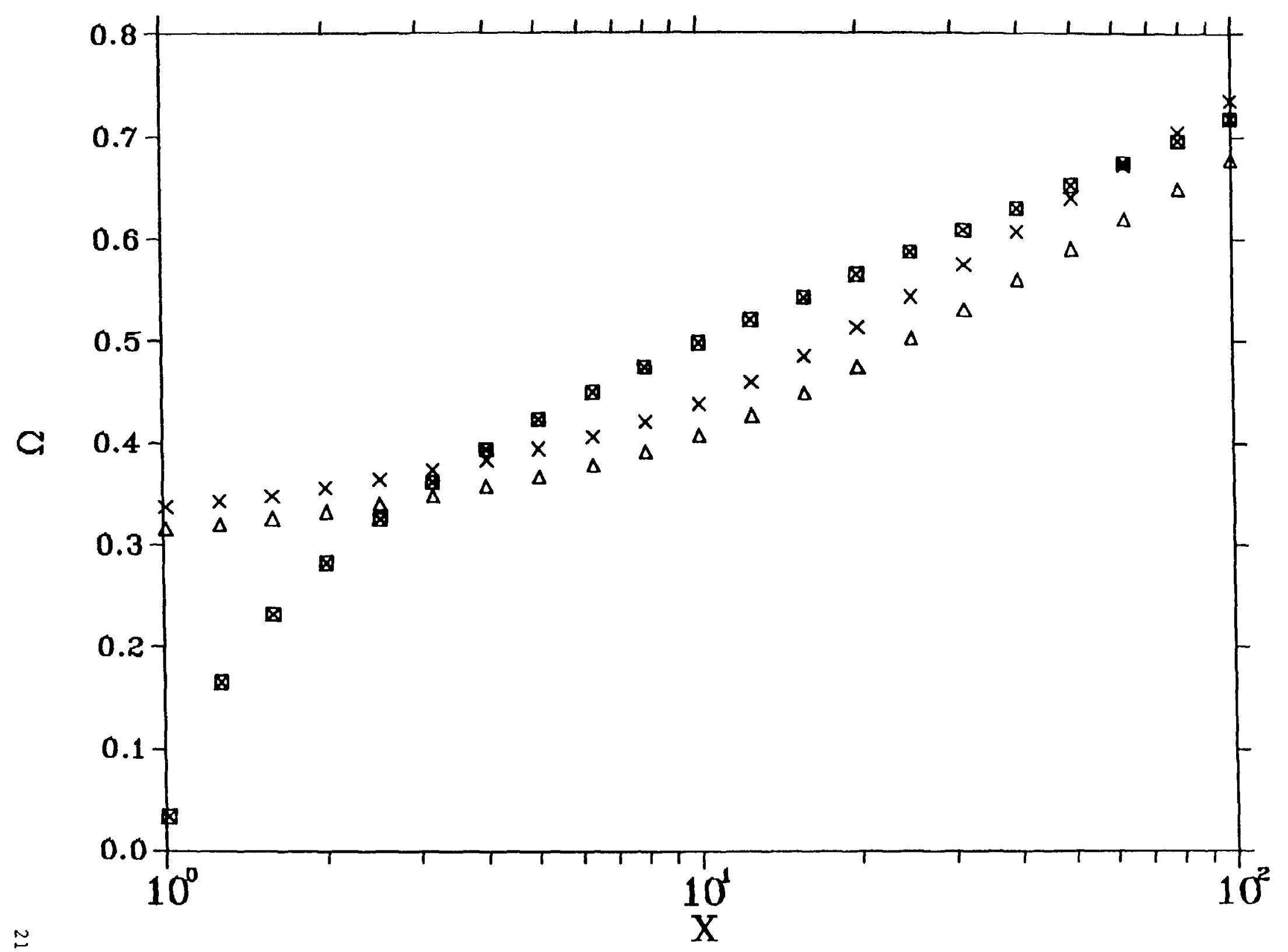

Fig. 9. Collision strengths $(\Omega)$ for the $2 s^{2}-2 s 2 p^{1} P$ transition in Fe XXIII. Triangles represent ACE DW-HF results; X's represent Coulomb-hydrogenic: results; boxes with $X$ 's represent PWB results. 


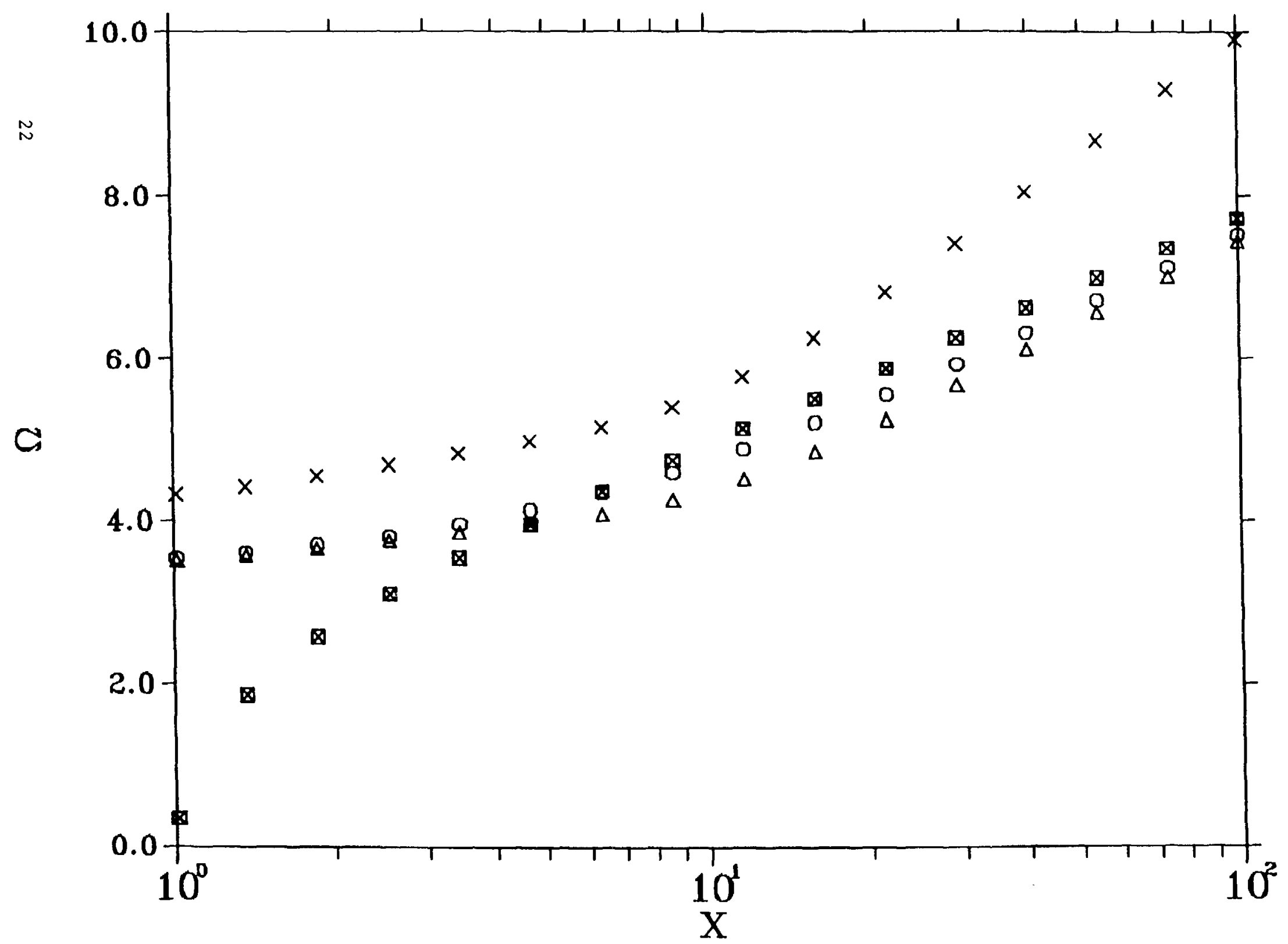

Fig. 10. Collision strengths $(\Omega)$ for the $3^{2} \mathrm{~S}-3^{2} \mathrm{P}$ transition in Fe XVI. Circles represent Mann's results; triangles are ACE DW-HF results; X's represent Coulombhydrogenic results; boxes witl X's are PWB result:- 


\section{CONCLUSION}

The ACE code is now available to produce electron impact excitation collision strengths. The code is easy to use and has a variety of options to allow comparison of results using different models. No large effort has yet been made to optimize the code: we have concentrated on getting this version working reliably on arbitrary transitions. In the near future, we plan to work on speeding up the code and adding options such as calculation of differential cross sections and polarization fractions.

\section{APPENDIX}

\section{INFORMATION CONTAINED ON THE PARADISE FILE FROM THE ACE CALCULATION}

Much of the information on the PARADISE file is copied from the structure file. In addition, collision strength information is written to the file. The following Table $A$ gives the set of key values and a brief description of the associated data. See Ref. 9 for a detailed description of the PARADISE format.

TABLE A

Key Values

$Z$, ion, seq, 'config', config number

$Z$, ion, seq, 'shellord'

Z, ion, seq, 'cfg'

$\mathrm{Z}$, ion, seq, ' $\mathrm{L}$ '

$Z$, ion, seq, 'S'

$Z$, ion, seq, ' $\mathrm{J}$ '

$Z$, ion, seq, ' $E$ '

$Z$, ion, seq, 'NOSUBC'

$Z$, ion, seq, 'SCRL'

$Z$, ion, seq, 'SCRS'
Description

Information on configurationoccupancy numbers and energies.

Information on the ordering of shells used in coupling the angular momentum.

Indexing of configuration going with each level of target.

Total $\mathrm{L}$ value for each level of target.

Total S value for each level of target.

Total $\mathrm{J}$ value for each level of target.

Energy for each level of target.

Maximum number of unfilled subshells.

Script L quantum numbers for each level.

Script S quantum numbers for each level. 


\section{TABLE A Cont'd.}

Key Values

$Z$, ion, seq, 'BLL'

$Z$, ion. seq, 'BLS'

$Z$, ion, seq, 'BLV'

$\mathrm{Z}$, ion, seq, 'MEIGV'

Z, ion, seq, 'LEVEIGV'

Z, ion, seq, 'EIGVEC', i

Z, ion, seq, 'LEVDW'

$Z$, ion, seq, 'LEVDWP'

$Z$, ion, seq, 'XDW'

$Z$, ion, seq, 'DWCS'
Description

Block L quantum numbers for each level.

Block S quantum numbers for each level.

Seniority quantum numbers for each level.

Number of levels in each group of mixed levels.

Listing of levels in each group of mixed levels.

Mixing coefficients for group $i$ of mixed levels.

Indices of initial levels for collision strengths.

Indices of final levels for collision strengths.

Energies in threshold units for collision strengths.

Collision strength values. 


\section{REFERENCES}

1. J. Abdallah, Jr., R. E. H. Clark, and R. D. Cowan, "Theoretical Atomic Physics Code Development I. CATS: Cowan Atomic Structure Code," (December 1988

2. R. E. H. Clark, J. Abdallah, Jr., and S. P. Kramer, "Theoretical Atomic Physics Code Development III. TAPS: A Display Code for Atomic Data," Los Alamos manual (in preparation).

3. J. Abdallah, Jr., R. E. H. Clark, and S. P. Kramer, "Theoretical Atomic Physics (TAPS) Code Development," Sixth APS Topical Conference on Atomic Processes in High Temperature Plasmas (Santa Fe, Sept. 1987).

4. J. B. Mann, At. Data Nuc. Data Tables, 29, 407 (1983).

5. G. Csanak, A. L. Merts, L. A. Collins, and J. B. Mann, Group T-4, Los Alamos National Laboratory (private communication).

6. G. Csanak, H. S. Taylor, and R. Yaris, Phys. Rev. A, 3, 1322 (1971).

7. G. Csanak, H. S. Taylor, and R. Yaris, Adv. At. Mol. Phys., Z, 287 (1971).

8. R. D. Cowan, Theory of Atomic Structure and Spectra, (University of California Press, Berkeley and Los Angeles, Calif., 1981).

9. J. Abdallah, Jr., and R. E. H. Clark, "Paradise Files," Los Alamos National Laboratory internal memorandum X-6:REHC-85-414 (Sept. 1986).

10. J. Abdallah, Jr., "User's Manual for CURVES," Los Alamos National Laboratory report LA-8844-M (1981).

11. M. Abramowitz and I. A. Stegun, Handbook of Mathematical Functions, (Dover Publications, NY, NY, 1972) p. 16.

12. H. E. Saraph, Comput. Phys. Commun., $\underline{3}, 256$ (1972).

13. R. E. H. Clark, Comput. Phys. Commun., 16, 119 (1978).

14. M. K. Inal and J. Dubau, J. Phys. B, 20, 4221 (1987).

15. S. W. White, Group X-6, Los Alamos National Laboratory (private communication). 